\title{
TET repression and increased DNMT activity synergistically induce aberrant DNA methylation
}

\author{
Hideyuki Takeshima, ${ }^{1}$ Tohru Niwa, ${ }^{1}$ Satoshi Yamashita, ${ }^{1}$ Takeji Takamura-Enya, ${ }^{2}$ Naoko lida, ${ }^{1}$ Mika Wakabayashi, \\ Sohachi Nanjo, ${ }^{3}$ Masanobu Abe, ${ }^{4,5}$ Toshiro Sugiyama, ${ }^{3}$ Young-Joon Kim, ${ }^{6}$ and Toshikazu Ushijima ${ }^{1}$
}

'Division of Epigenomics, National Cancer Center Research Institute, Tokyo, Japan. ${ }^{2}$ Department of Applied Chemistry, Kanagawa Institute of Technology, Kanagawa, Japan. ${ }^{3}$ Third Department of Internal Medicine, University of Toyama, Toyama, Japan. ${ }^{4}$ Department of Oral and Maxillofacial Surgery, University of Tokyo Hospital, Tokyo, Japan. ${ }^{5}$ Division for Health Service Promotion, University of Tokyo, Tokyo, Japan. ${ }^{6}$ Department of Biochemistry, College of Life Science and Biotechnology, Yonsei University, Seoul, Korea.

\begin{abstract}
Chronic inflammation is deeply involved in various human disorders, such as cancer, neurodegenerative disorders, and metabolic disorders. Induction of epigenetic alterations, especially aberrant DNA methylation, is one of the major mechanisms, but how it is induced is still unclear. Here, we found that expression of TET genes, methylation erasers, was downregulated in inflamed mouse and human tissues, and that this was caused by upregulation of TET-targeting miRNAs such as MIR20A, MIR26B, and MIR29C, likely due to activation of NF- KB signaling downstream of IL-1 $\beta$ and TNF- $\alpha$. However, TET knockdown induced only mild aberrant methylation. Nitric oxide (NO), produced by NOS2, enhanced enzymatic activity of DNA methyltransferases (DNMTs), methylation writers, and NO exposure induced minimal aberrant methylation. In contrast, a combination of TET knockdown and NO exposure synergistically induced aberrant methylation, involving genomic regions not methylated by either alone. The results showed that a vicious combination of TET repression, due to NF- $\kappa B$ activation, and DNMT activation, due to NO production, is responsible for aberrant methylation induction in human tissues.
\end{abstract}

\section{Introduction}

Chronic inflammation is deeply involved in various human chronic disorders, such as cancer (1-3), neurodegenerative disorders (4), diabetes mellitus (5), and osteoarthritis (6). Induction of epigenetic alterations is considered to be one of the major mechanisms. Specifically, DNA methylation of promoter $\mathrm{CpG}$ islands of tumor suppressor genes such as BRCA1, CDH1 (E-cadherin), CDKN2A (p16), and $R B$ is known to be involved in a variety of cancer types (7-10). In human life, aberrant DNA methylation is induced in normal tissues in the very early stages of cancer development, and the degree of methylation accumulation (methylation burden) in normal tissues is correlated with cancer risk, forming a field for cancerization (11-13). The impact of methylation burden on cancer risk was first demonstrated by cross-sectional studies (14), and is now also demonstrated by a multicenter prospective cohort clinical study $(15,16)$.

Aberrant DNA methylation is induced by aging (17-19) and also by exposure to various environmental stimuli, such as infectious agents (20), oxidative stress (21), hormone exposure (22, 23), and smoking (24). Infectious agents are known to induce aberrant DNA methylation via chronic inflammation, such as gastritis triggered by Helicobacter pylori (H. pylori) infection $(25,26)$, hepatitis triggered by hepatitis virus (HBV and HCV) infection $(27,28)$, and cholangitis triggered by liver fluke (29). Importantly, the expression levels of specific inflammationrelated genes, $I l 1 b$, Nos2, and $\operatorname{Tnf}$, have been shown to correlate

Conflict of interest: The authors have declared that no conflict of interest exists. Copyright: () 2020, American Society for Clinical Investigation.

Submitted: August 7, 2018; Accepted: July 7, 2020; Published: September 14, 2020.

Reference information: J Clin Invest. 2020;130(10):5370-5379.

https://doi.org/10.1172/JCI124070. with the degree of aberrant methylation induction in multiple tissues $(26,30,31)$, suggesting that signaling pathways regulated by these genes are involved in methylation induction. However, mechanisms of how these inflammation-related genes are involved in induction of aberrant DNA methylation, especially how writers (DNA methyltransferases [DNMTs]) and erasers (TET methylcytosine dioxygenases [TETs]) of DNA methylation are dysregulated, are mostly unknown.

In this study, we aimed to clarify the mechanisms of methylation induction in epithelial cells by exposure to chronic inflammation. A mouse gastritis model triggered by Helicobacter felis (H.felis) infection was used to identify molecular changes with in vivo relevance. Molecular analyses of the writers and erasers and their functional impact were complemented by using engineered human cell lines.

\section{Results}

Chronic inflammation by $H$. felis is capable of inducing aberrant DNA methylation. Induction of chronic inflammation in mouse gastric tissues by $H$. felis infection was initially confirmed (Figure 1A). Hyperplastic changes with infiltration of inflammatory cells, predominantly mononuclear cells, were observed in gastric tissues at 40 weeks of $H$. felis infection (Figure 1B), confirming the presence of chronic inflammation. Il1b, Nos2, and Tnf, whose expression levels correlate with induction of aberrant DNA methylation in human and gerbil stomachs (refs. 26, 30, 31 and Supplemental Figure 1A; supplemental material available online with this article; https://doi. org/10.1172/JCI124070DS1), were also upregulated in mouse gastric tissues infected with $H$. felis (Figure $1 \mathrm{C}$ ). Activation of the NF- $\mathrm{KB}$ signaling pathway, a downstream pathway of Il-1 $\beta$ and Tnf- $\alpha$, was confirmed by increased levels of the phosphorylated form of RelA 


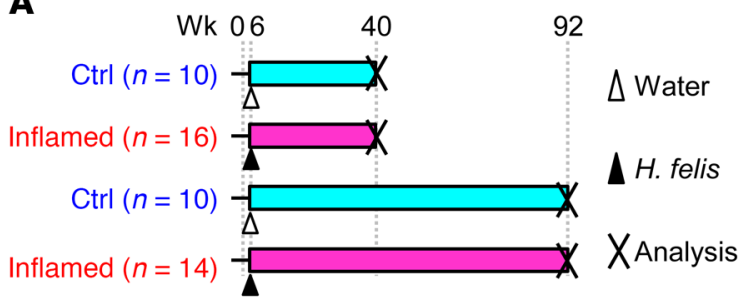

B

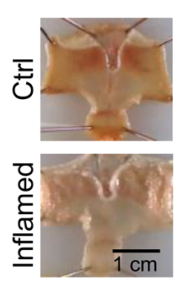

40 wk

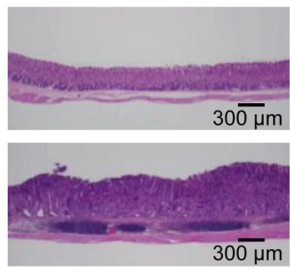

C
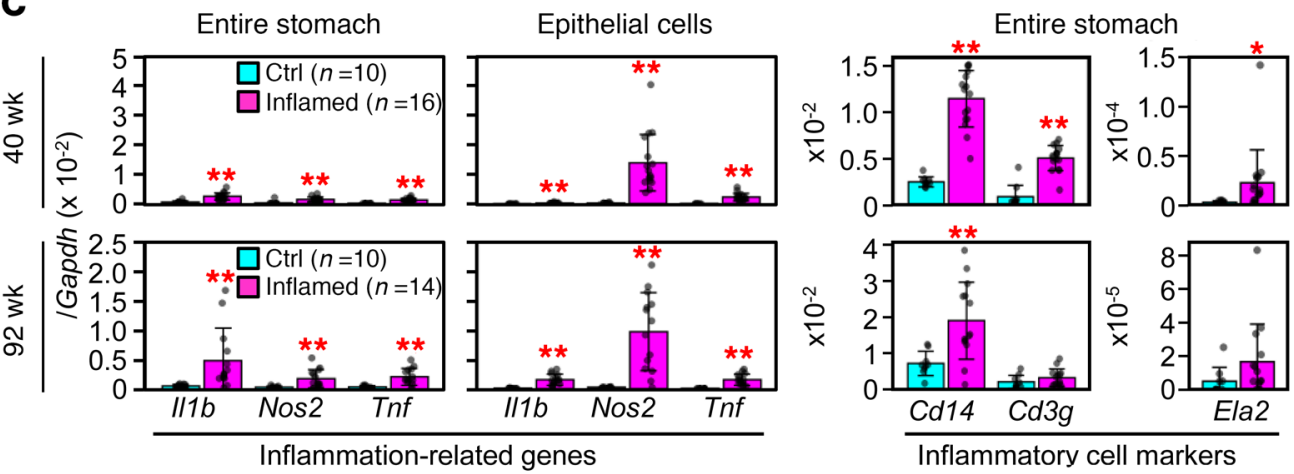

D

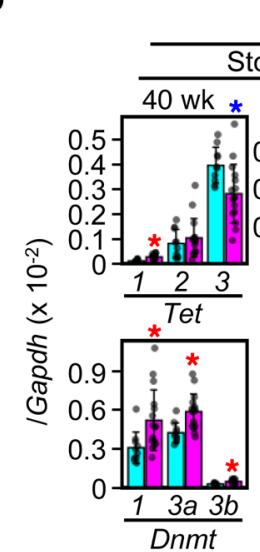

Ctrl $\square(n=10)$ Inflamed $\square(n=16)$
Mouse
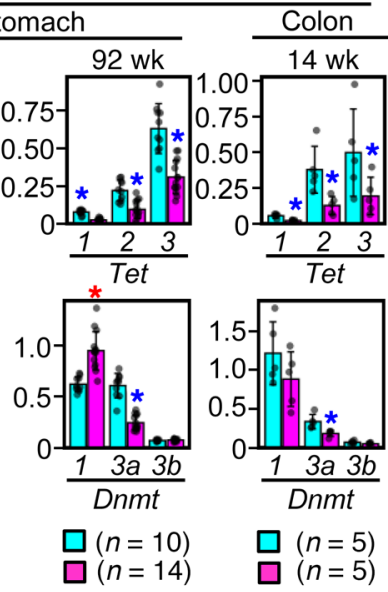

$\square(n=14)$ $\square(n=5)$
$\square(n=5)$
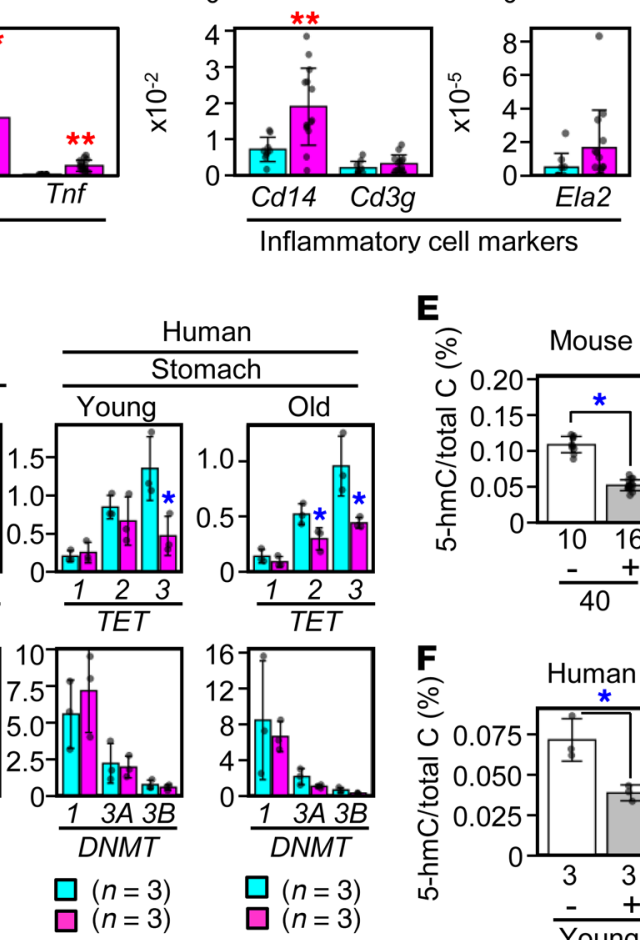

Inflammatory cell markers

Figure 1. Tet repression by exposure to chronic inflammation. (A) Experimental protocol of $H$. felis infection. Mice were infected with $H$. felis for 34 weeks (40 weeks of age) or 86 weeks (92 weeks of age). Inflamed, H. felis-infected; Ctrl, mock-treated. (B) Histological changes in the stomach by H. felis infection. Hyperplastic changes and infiltration of inflammatory cells were observed by $H$. felis infection. Inflamed, H. felis-infected; Ctrl, mock-treated. (C) mRNA expression changes of inflammation-related genes by exposure to chronic inflammation. I/1b, Nos2, and Tnf were upregulated by exposure to chronic inflammation. Inflamed, $H$. felis-infected (40 weeks, $n=16$; 92 weeks, $n=14$ ); Ctrl, mock-treated (40 weeks, $n=10 ; 92$ weeks, $n=10$ ). Data represent mean $\pm \mathrm{SD}$ (Welch's $t$ test, ${ }^{*} P<0.05$; ${ }^{* *} P<0.01$ ). (D) mRNA expression changes of the Tet and Dnmt genes by exposure to chronic inflammation. Tet3 was mildly repressed at 40 weeks of age, and all 3 Tet genes were repressed at 92 weeks of age. On the other hand, Dnmt expression did not show major changes. The stomachs of $H$. felis-infected mice at 40 weeks (inflamed, $n=16 ; \mathrm{ctrl}, n=10$ ) and 92 weeks (inflamed, $n=14 ; \mathrm{ctrl}, n=10$ ), the colons of DSS-treated mice (inflamed, $n=5$; ctrl, $n=5$ ), and the stomachs of $H$. pylori-infected humans (inflamed, $n=3 ; c t r l, n=3$ for both young and old) were analyzed. Data represent mean \pm SD (Welch's $t$ test, ${ }^{*} P<0.05$ ). (E and F) The 5-hmC content was measured by LC/MS/MS combined with HPLC. The 5-hmC content in genomic DNA was reduced both in the mouse stomach (40 weeks: inflamed, $n=16$; ctrl, $n=10$; 92 weeks: inflamed, $n=14 ; \mathrm{ctrl}, n=10$ ) (E) and the human stomach (young: inflamed, $n=3$, ctrl, $n=3$; old: inflamed, $n=3$, ctrl, $n=3$ ) (F) by exposure to chronic inflammation. Data represent mean \pm SD (Welch's $t$ test, $\left.{ }^{*} P<0.05\right)$.

protein (Supplemental Figure 1B) and the increased expression of a downstream target gene, Ccl2 (Supplemental Figure 1C).

Genomic regions with aberrant DNA methylation were searched for by MBD-seq of gastric epithelial cells of 3 control and $3 \mathrm{H}$. felis-infected mice. Among the 28,761 promoter regions, 26,603 regions were commonly unmethylated in 3 control mice
(Supplemental Figure 2A), and 215, 176, and 287 regions were hypermethylated (aberrantly methylated) in the 3 inflamed mice, respectively. One hundred thirty-eight regions were commonly hypermethylated in 2 or 3 mice (Supplemental Figure 2B) and a tumor suppressor gene, Ajap1 (32), was among them (Supplemental Figure 2, C and D). A similar degree of overlap was observed for 
hypomethylated regions (Supplemental Figure 2E). These results showed that chronic inflammation characterized by upregulation of $I l 1 b$, Nos2, and Tnf, and aberrant DNA methylation, were induced in gastric tissues of $H$. felis-infected mice.

Tet genes are repressed by exposure to chronic inflammation. To explore the mechanisms of methylation induction by exposure to chronic inflammation, we first analyzed expression changes of DNA methylation writer (Dnmt) and eraser (Tet) genes in mouse gland-isolated gastric epithelial cells, mouse glandisolated colon epithelial cells (31), gerbil gland-isolated gastric epithelial cells, and human gastric tissues, all of which were with and without inflammation. Regarding Dnmt genes, Dnmt1 expression increased by chronic inflammation in the mouse gastric epithelial cells, but not in the mouse colon epithelial cells or human gastric tissues (Figure 1D).

In contrast, Tet 3 was consistently repressed in mouse gastric epithelial cells, mouse colon epithelial cells, gerbil gastric epithelial cells, and human gastric tissues with inflammation (Figure 1D and Supplemental Figure 3). In addition to Tet3, Tet1 and Tet2 were also repressed in mouse gastric epithelial cells with long-term exposure to inflammation (92 weeks). The content of 5-hydroxymethyl-2'deoxycytidine $(5-\mathrm{hmC})$ was reduced to less than half in inflamed mouse gastric epithelial cells and human gastric tissues (Figure 1, $\mathrm{E}$ and $\mathrm{F}$ ), supporting the biological significance of Tet repression. These results showed that Tet genes were markedly repressed by exposure to chronic inflammation, such as $H$. felis-triggered mouse gastritis, dextran sulfate sodium-triggered (DSS-triggered) mouse colitis, and H.pylori-triggered gerbil and human gastritis.

Upregulation of specific miRNAs represses Tet genes. To identify the molecular mechanism of Tet repression by chronic inflammation, we analyzed the possibility of induction of Tet-targeting miRNAs based upon previous reports $(33,34)$. Expression analysis of 1,881 miRNAs in gastric epithelial cells from 4 control and $4 \mathrm{H}$. felisinfected mice revealed that 36 miRNAs were upregulated 5-fold or more in inflamed gastric epithelial cells (Figure 2A and Supplemental Figure 4). At the same time, in silico analysis predicted that 16, 67, and 51 miRNAs can potentially target Tet1, Tet2, and Tet3, respectively (Figure 2B). Combining the data, 12 miRNAs were considered to target at least 1 of the 3 Tet genes, and were upregulated by chronic inflammation (Figure 2C). Among the 12 miRNAs considered, 6 (miR-29c, miR-26a, miR-26b, miR-20a, miR-20b, and miR-106b) were predicted to target multiple Tet genes in both mice and humans (Figure 2C and Supplemental Figure 5), due to having multiple target sites at the 3 '-UTR regions (Figure 2D).

TET-targeting activity of 4 (MIR29C, MIR26B, MIR20A, and MIR2OB) of the 6 miRNAs was experimentally analyzed by introducing their mimics into cultured 293FT and MCF7 cells and measuring expression of the 3 TET genes. 293FT and MCF7 cells were used, as they had high transfection efficiencies and miRNA target sequences were unlikely to be affected by cellular contexts. MIR29C consistently repressed the 3 TET genes in the 2 cell lines, MIR26B repressed TET3 in the 2 cell lines, and MIR20A mildly repressed TET3 only in 293FT cells (Figure 2E). In contrast, MIR20B did not repress expression of any of the 3 TET genes (data not shown). The influence of the 3 miRNAs (MIR29C, MIR26B, and MIR20A) on the 5 -hmC content was examined by transfecting 293FT cells with one of the miRNAs or their combination. The content was reduced to below the detection limit by MIR29C, MIR26B, and the combination of all the 3 miRNAs, and to $58.1 \%$ by MIR20A (Supplemental Figure 6).

Among the 3 miRNAs, MIR26B was also upregulated in inflamed human gastric tissues (Supplemental Figure 7). The expression level of MIR26B was negatively correlated with that of TET3 both in human and mouse gastric epithelial cells (Supplemental Figure 8). TET3 had 2 target sites of MIR26B in its 3'-UTR region (Supplemental Figure 9 and Figure 2F). Luciferase introduced with the target sites showed only half the activity in the presence of MIR26B (Figure 2G), but the reduction was canceled by the introduction of point mutations into the 2 MIR26B target sites (Figure 2G).

Upregulation of TET-targeting miRNAs is likely to be mediated by NF- $\kappa B$ activation. To analyze the mechanism of the upregulation of MIR29C, MIR26B, and MIR2OA, we focused on the NF- $\mathrm{BB}$ signaling pathway. The 3 miRNAs were expected to be cotranscribed along with their host genes, respectively $(35,36)$, and both H3K4me3 and H3K27Ac were enriched around the promoter regions of the host genes in 7 cell lines from the Encyclopedia of DNA Elements (ENCODE) (Supplemental Figure 10). Therefore, we conducted ChIP-seq analysis of a human gastric cancer cell line, NUGC-3, treated with TNF- $\alpha$ to analyze the binding status of NF- $\kappa \mathrm{B}$ subunit RELA (p65), around the promoter regions of the 3 host genes. NF- $\kappa \mathrm{B}$ activation by TNF- $\alpha$ was confirmed by increased expression of a downstream target gene, IL6 (Figure $3 \mathrm{~A})$. Peaks with peak scores greater than 6.0 were detected in NUGC- 3 cells treated with mock (1x PBS (-)) $(n=2739)$ and TNF- $\alpha$ ( $n=19,206)$ (Figure 3B and Supplemental Tables 1 and 2). NF-kB binding motifs were most significantly enriched among the peaks detected in NUGC-3 cells treated with TNF- $\alpha$ (Figure 3C), showing successful detection of RELA binding sites. RELA binding levels at the putative promoter regions of MIR26B (CTDSP1) and MIR20A (MIR17HG) robustly increased by TNF- $\alpha$ treatment (Figure 3, $\mathrm{D}$ and $\mathrm{E}$ ), but the RELA binding level at the putative promoter region of MIR29C (C1orf132) did not (Supplemental Figure 11). The expression level of MIR17HG was accordingly upregulated by TNF- $\alpha$ treatment (Supplemental Figure 12). RELA binding levels at the host genes were comparable to the binding level at the IL6 promoter (Figure 3F).

The effects of NF- $\kappa \mathrm{B}$ inhibition on the expression levels of the 3 miRNAs and their host genes were further analyzed in 293FT cells, in which RELA was phosphorylated even without TNF- $\alpha$ treatment. NF- $\kappa$ B inhibition by BAY 11-7082 was confirmed by the decrease of the phosphorylated form of RELA protein (Supplemental Figure 13A). The expression levels of CTDSP1 and MIR17HG were downregulated in a dose-dependent manner (Supplemental Figure 13B), but those of MIR26B and MIR20A were not (Supplemental Figure 13C). The discrepancy between the host gene and miRNA expression could be explained by higher stability of miRNAs than messenger RNAs (mRNAs) (37). The results further supported that some TETtargeting miRNAs were likely to be upregulated by the activation of the NF- $\mathrm{KB}$ signaling pathway.

Nitric oxide exposure enhanced DNMT activity. In addition to $I l 1 b$ and Tnf, Nos 2 expression, involved in the production of nitric oxide (NO), has been consistently associated with aberrant 
A

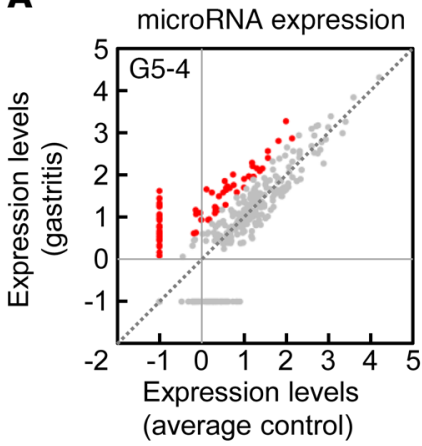

B

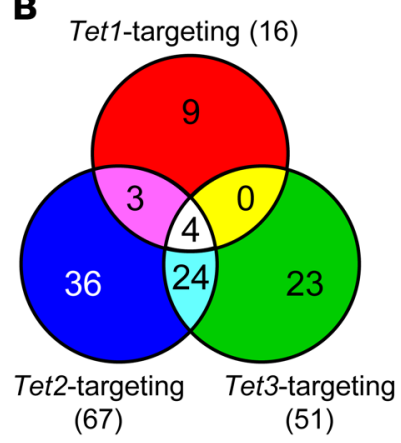

C

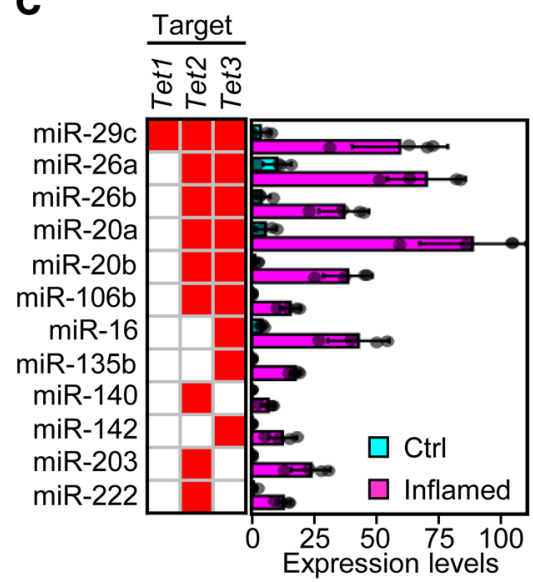

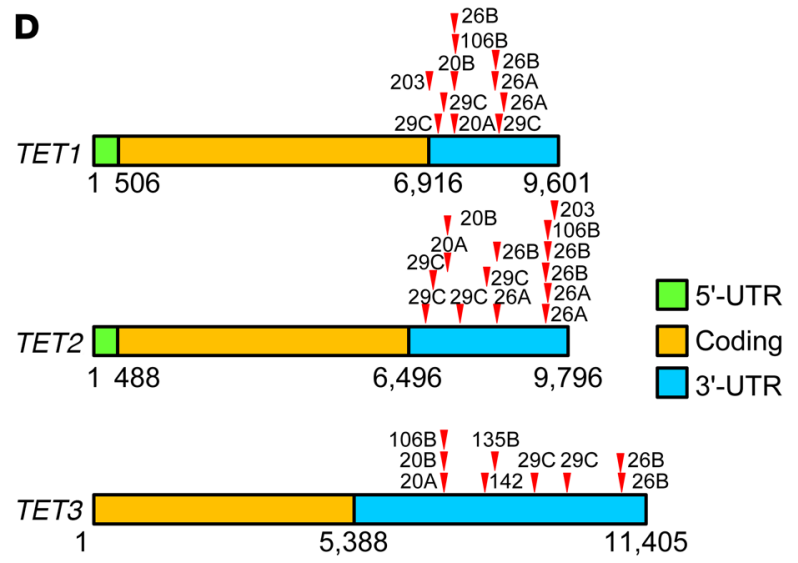

$\mathbf{F}$

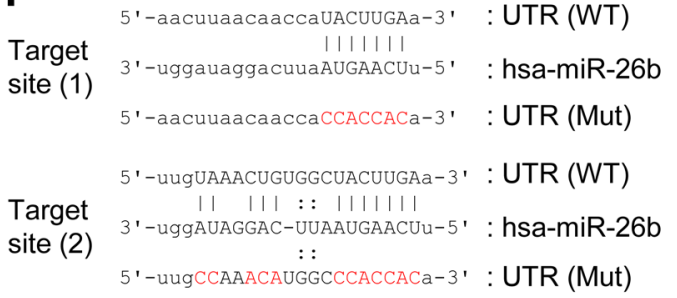

E

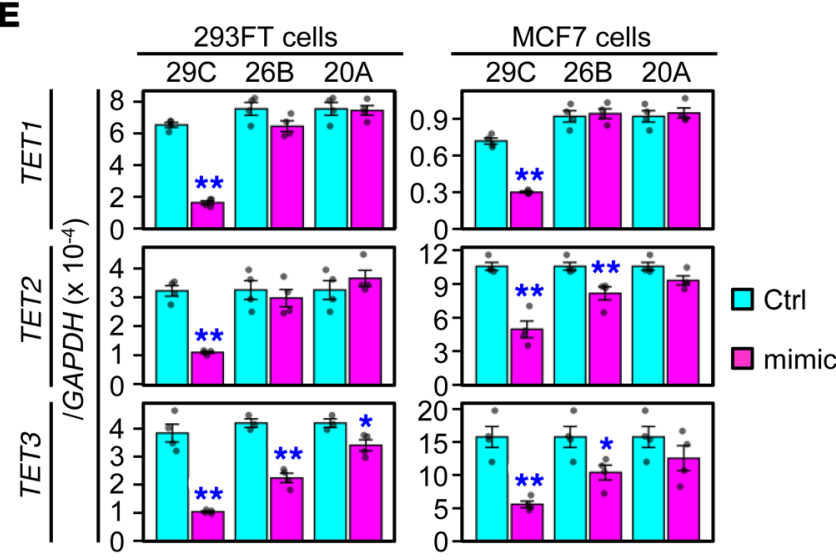

G

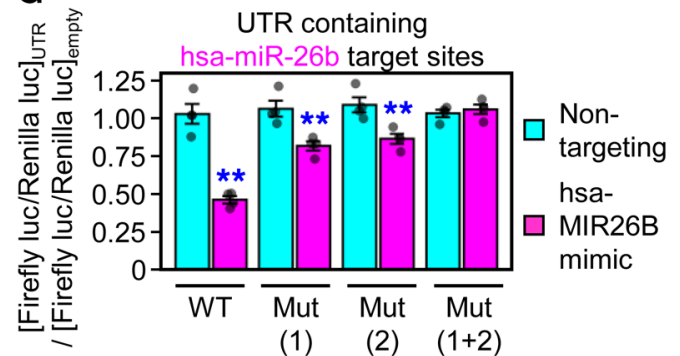

Figure 2. Upregulation of Tet-targeting miRNAs by exposure to chronic inflammation. (A) miRNA upregulated by exposure to chronic inflammation in a mouse (G5-4). Thirty-six miRNAs were upregulated 5-fold or more by exposure to chronic inflammation. (B) In silico prediction of Tet-targeting miRNAs. Sixteen, 67, and 51 miRNAs were predicted to target Tet1, Tet2, and Tet3, respectively. (C) Identification of Tet-targeting miRNAs upregulated by the exposure to chronic inflammation. Twelve miRNAs that can potentially target one or more Tet genes (shown by red squares) were upregulated by exposure to chronic inflammation in 4 mice. Data represent mean $\pm S D(n=4)$. (D) Potential target sites for the miRNAs in the $3^{\prime}-$ UTR regions of the TET genes. Some miRNAs had multiple target sites in a single TET gene. (E) Repression of TET genes by MIR29C, MIR26B, and MIR20A. Introduction of these miRNAs into 293FT cells repressed the expression of the TET genes. Data represent mean \pm SE (Welch's $t$ test, ${ }^{*} P<0.05 ;{ }^{* *} P<0.01$ ). (F) MIR26B target sites within the $3^{\prime}$-UTR region of human TET3. The $3^{\prime}$-UTR regions with WT sequences (WT) and sequences with 1 mutation or 2 mutations (Mut) were cloned into a reporter vector. (C) Luciferase assay using the $3^{\prime}$-UTR region of human TET3. Reduction of the luciferase activity by the introduction of MIR26B mimic was canceled by introduction of the 2 mutations into the target sites. Data represent mean \pm SE (Welch's $t$ test, ${ }^{* *} P<0.01$ ).

DNA methylation induction $(26,30,31)$. Based on the reports that exposure of nuclear extract to NO enhanced the DNMT enzymatic activity $(38,39)$, nuclear proteins extracted from gastric cancer cell lines HSC41 and TMK1 were treated with NO donors NOC18 or SNAP. The enzymatic activity of DNMTs was confirmed to be enhanced by both NOC18 (3.3- to 4.8-fold) and SNAP (1.4- to 1.5-fold) (Supplemental Figure 14). In contrast, expression levels of all the 3 TET genes were not repressed and those of MIR26B were not upregulated by treatment with NO (Supplemental Figure 15). These results confirmed that NO exposure enhanced DNMT activity.

Synergistic effect of a combination of TET repression and increased DNMT activity. To examine the effect of TET repression and NOC18 treatment on aberrant DNA methylation induction, TET3, 
most abundantly expressed in the stomach, was repressed by a short hairpin RNA (shRNA) in 293FT cells (Supplemental Figure 16). $293 \mathrm{FT}$ cells were used since $\mathrm{CpG}$ islands susceptible to methylation induction are already methylated in cancer cell lines. Cells were cultured for 4,10 , and 20 weeks because sufficient exposure time to chronic inflammation was known to be needed for methylation induction in vivo (26). DNA methylation induction was analyzed by a DNA methylation microarray, which is known to detect DNA methylation accurately in human cells $(40,41)$.

After a 4-week culture, TET3 knockdown alone induced aberrant DNA methylation $(\Delta \beta \geq 0.2)$ at only a small number of genomic blocks (genomic regions within $500 \mathrm{bp}$; a total of 535,684 genomic blocks in the genome [ref. 42]) (3568; 0.67\%). NOC18 treatment alone induced aberrant methylation at a limited number of genomic blocks (3158; 0.59\%). In contrast, their combination induced aberrant DNA methylation at a larger number of genomic blocks $(15,658 ; 2.92 \%)$ (Figure $4 \mathrm{~A})$. When culture periods were extended, the numbers of methylated blocks increased, especially those methylated by the combination (61,964 [11.57\%] at 20 weeks) (Figure 4A). At 4 and 10 weeks, 2 more independent cultures were analyzed: for TET3 knockdown, using 2 additional shRNAs, and for NOC18 treatment, using 2 more biological replicates. The combination of TET3 knockdown by additional shRNAs and NOC18 treatment was also analyzed (Supplemental Figure 17). It was confirmed that similar numbers of genomic blocks were methylated, and the combination had strong effects.

Using the methylation data from a total of 3 independent cultures at 4 and 10 weeks, a volcano plot analysis was conducted. The number of genomic blocks with larger $\Delta \beta$ values and larger $-\log _{10}$ $($ FDR $q$ ) values greatly increased in 293FT cells treated with the combination (Figure $4 \mathrm{~B}$ ). $\Delta \beta$ values became much larger in $293 \mathrm{FT}$ cells treated with the combination at 10 weeks. However, $-\log _{10}($ FDR q) values at 10 weeks became smaller, due to the culture perioddependent increases in the noise of methylation levels (in control cultures, $\mathrm{SD}=0.018 \pm 0.016$ at 4 weeks; $0.024 \pm 0.020$ at 10 weeks). These results showed that aberrant DNA methylation was strongly induced by the combination of TET repression and NO exposure.

Biological relevance of aberrantly methylated blocks by the combination. To examine the biological relevance of DNA methylation induced by the combination, a gastric cancer cell line, HSC60, was additionally analyzed (Figure 5A and Supplemental Figure 18). The numbers of methylated genomic blocks were smaller than those in 293FT cells, as expected. However, the combination effect was clearly observed also in the HSC60 cells (Figure 5A). After a 20-week culture, 15,007 genomic blocks were aberrantly methylated by the combination, and 8596 of them were not methylated $(\Delta \beta<0.2)$ by TET3 knockdown alone or NOC18 treatment alone. Using these 8596 blocks, the nature of genomic blocks methylated by the combination was examined. Most of the blocks hypermethylated by the combination were located in gene body regions without $\mathrm{CpG}$ islands (Figure 5B). Twenty genomic blocks were located within promoter $\mathrm{CpG}$ islands, and biological relevance of the 20 promoter $\mathrm{CpG}$ islands was analyzed by gene ontology. Genes involved in responses to external stimulus, regulation of secretion, and cellular homeostasis were enriched (Supplemental Table 3). Importantly, these genes were also methylated in primary noncancerous tissues of gastric cancer patients (Figure 5C).

\section{Discussion}

A combination of TET repression, due to NF- $\mathrm{BB}$ activation, and increased DNMT activity, due to exposure to NO, had a synergistic effect in aberrant DNA methylation induction (Figure 5D). Therefore, this vicious combination was considered to be important for aberrant DNA methylation induction in $\mathrm{H}$. felis- and $\mathrm{H}$. pyloritriggered gastritis, and also chronic inflammation in other tissues with NF- $\mathrm{BB}$ activation and increased NO production, such as liver tissues exposed to hepatitis virus (HBV and HCV) $(27,28)$, colon tissues exposed to ulcerative colitis (17), and Barrett's esophagus (43). In addition to cancers, it has been reported that IL-1 $\beta$ and NO levels are increased in neuroinflammation associated with neurodegenerative disorders and psychiatric disorders, osteoarthritis, and obesity (44-46). This suggests that the vicious combination may underlie various disorders in addition to cancers. The combination may be present in tissues even with little histologically identifiable inflammation. A combined administration of NF- $\kappa \mathrm{B}$ inhibitor and NO antagonist might have potent effects for cancer prevention, and its usefulness needs to be addressed using an animal model in which both NF- $\mathrm{kB}$ activation and NO production are present.

In previous studies, induction of aberrant DNA methylation by NO was observed only for a single gene, mostly by nonquantitative methods. FMR1 was methylated in Jurkat T cells exposed to NO donors SIN-1 or SNAP (38), and CDH1 was methylated in gastric cancer cells exposed to NO produced by IL-1 $\beta$ treatment (39). In this study, aberrant DNA methylation induction was analyzed in a genome-wide manner using a highly quantitative microarray in cells treated for as long as 20 weeks. Nevertheless, aberrant DNA methylation was induced only at minimal numbers of genomic blocks, showing that exposure to NO has only a limited capacity of inducing aberrant DNA methylation but that a vicious combination is biologically important. As for the mechanism of enhancement of DNMT activity by NO, involvement of nitrosation of cysteine residues of DNMT proteins themselves or their regulators has been suggested (38).

Not only hypermethylation but also hypomethylation were observed in gastric epithelial cells of $\mathrm{H}$. felis-infected mice. In general, both regional hypermethylation (aberrant DNA methylation) and global hypomethylation, especially at repetitive elements $(2,3,47)$, are present in cancer cells, and our finding in mice was in line with humans exposed to H. pylori-triggered chronic inflammation (48). As for the mechanism of hypomethylation induction, it is possible that maintenance DNA methylation could become insufficient due to increased cell proliferation (49). However, our study focused on the mechanism of regional hypermethylation between the 2 methylation changes; the mechanism of global hypomethylation needs further investigation. In conclusion, a vicious combination of TET repression and increased DNMT activity had a synergistic effect on induction of aberrant DNA methylation.

\section{Methods}

Further information can be found in the Supplemental Methods.

Animal experiments. Mouse gastritis was induced by inoculating H. felis (ATCC 49179, ATCC) into 6-week-old male C57BL/6J mice (CLEA Japan). After 34 or 86 weeks of infection, mice were sacrificed and the stomach was resected. Mouse colitis was induced in 6-week- 

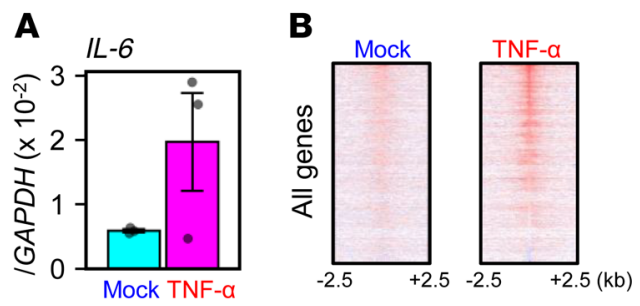

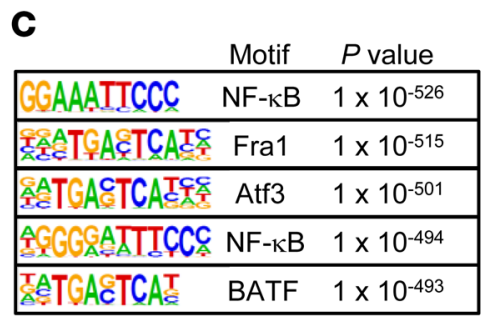
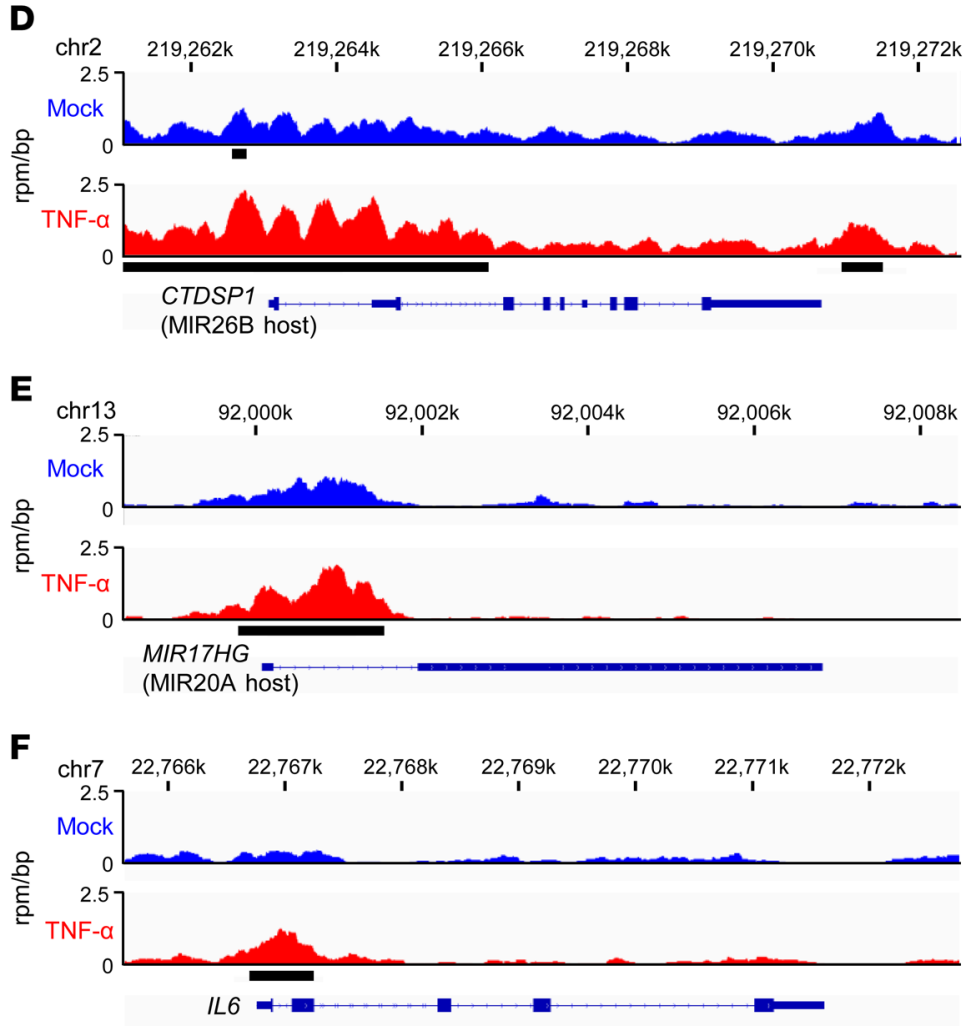

Figure 3. Increased RELA binding levels at promoter regions of TET-targeting miRNAs. (A) Activation of NF- $\kappa B$ signaling pathway in NUGC-3 cells by TNF- $\alpha$. A downstream target gene of NF- $\kappa B$ signaling pathway, IL6, was upregulated by TNF- $\alpha$ treatment. Data represent mean $\pm \mathrm{SE}$. (B) Heatmap of RELA binding levels in NUGC-3 cells treated with TNF- $\alpha$. RELA binding levels at genomic regions around TSSs of 44,112 transcripts were aligned according to the binding level after TNF- $\alpha$ treatment. Clear increase by the treatment was observed. Each row shows $\pm 2.5 \mathrm{~kb}$ centered on TSS. (C) Enriched motifs in RELA peaks detected in NUGC- 3 cells treated with TNF- $\alpha$. NF- $\kappa B$ binding motifs were most significantly enriched in NUGC-3 cells treated with TNF- $\alpha$, showing successful detection of RELA binding sites. (D-F) RELA binding status around the putative promoter regions of TET-targeting miRNAs. RELA binding levels at putative promoter regions of MIR26B (CTDSP1) (D) and MIR20A (MIR17HG) (E) were robustly increased by TNF- $\alpha$ treatment. RELA binding levels at these host genes were comparable to that at the ILG promoter $(\mathbf{F})$. Black boxes indicate genomic regions with peaks detected. The $y$ axis represents the read pileup normalized to the total number of reads at a base pair position ( $\mathrm{rpm} / \mathrm{bp}$ ). old male BALB/c mice (Charles River Laboratories) by administration of $2 \%$ DSS (molecular weight $=36,000-50,000$ ) as described (31). After 14 weeks, mice were sacrificed and the colon was resected.

Gastric and colon epithelial cells were isolated from the gastric glands and colonic crypts, respectively, as described (50), and used for analysis of epithelial cell-specific methylation and expression changes. Gastric and colon tissue containing both mucosal and muscle layers was used for expression analysis of inflammation-related genes. For histological analysis, whole mouse stomach was fixed by formalin and embedded in paraffin. The formalin-fixed paraffin-embedded (FFPE) samples were sliced and stained with hematoxylin and eosin.

Cell culture. The 293FT cell line was purchased from Thermo Fisher Scientific, the MCF7 cell line was purchased from the American Type Culture Collection, and the NUGC-3 cell line was purchased from Japanese Collection of Research Bioresources. The 3 gastric cancer cell lines were provided by K. Yanagihara (National Cancer Center, Tokyo, Japan) (HSC41 and HSC60) and W. Yasui (Hiroshima University, Hiroshima, Japan) (TMK1). The absence of Mycoplasma infection was confirmed using the MycoAlert mycoplasma detection kit (Lonza). 293FT cells were maintained in DMEM containing 10\% (vol/vol) FBS. MCF7, NUGC-3, HSC41, and TMK1 cells were maintained in RPMI1640 containing 10\% (vol/vol)
FBS. HSC60 cells were maintained in RPMI1640 with high glucose containing $10 \%$ (vol/vol) FBS.

Clinical samples. Twelve normal gastric tissue samples (6 samples infected with $H$. pylori and 6 noninfected samples) were endoscopically collected from healthy volunteers and stored in RNAlater (Thermo Fisher Scientific) at $-80^{\circ} \mathrm{C}$. All the gastric tissue samples were collected with written informed consent.

DNA methylation analysis. DNA methylation microarray analysis of human cells was performed using an Infinium MethylationEPIC BeadChip Kit (Illumina), which is highly reproducible for genome-wide DNA methylation analysis but only available for human cells $(40,41)$ as described (42). A total of $851,494 \mathrm{CpG}$ sites (probes) was assembled into 551,478 genomic blocks (assemblies of $\mathrm{CpG}$ sites) that were classified according to their relative locations (a) from a transcription start site (TSS) and (b) against a CpG island. Among the 548,543 genomic blocks, 535,684 blocks were located on autosomes; these were used for the analysis. DNA methylation levels ( $\beta$ values) of individual genomic blocks were evaluated using the mean $\beta$ values of all the probes within individual genomic blocks (51). Genomic blocks with DNA methylation levels increased at $20 \%$ or more $(\Delta \beta \geq 0.2)$, which was larger than the biological fluctuation, were defined as methylated blocks. DNA 
A

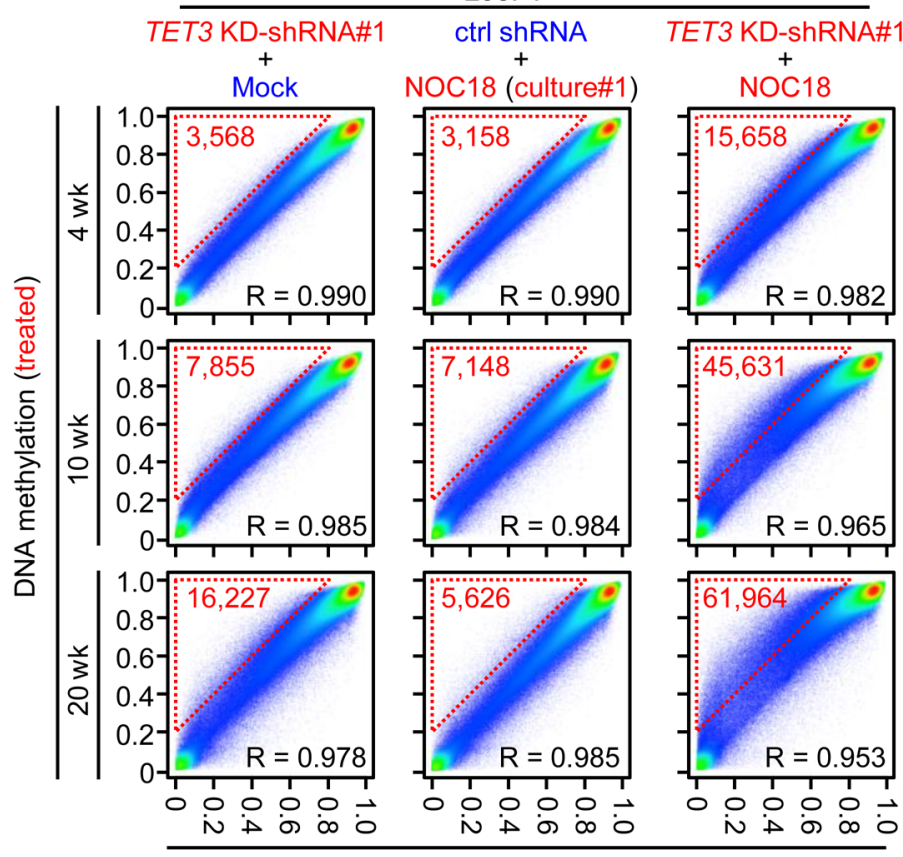

DNA methylation (ctrl shRNA + mock)

\section{B}

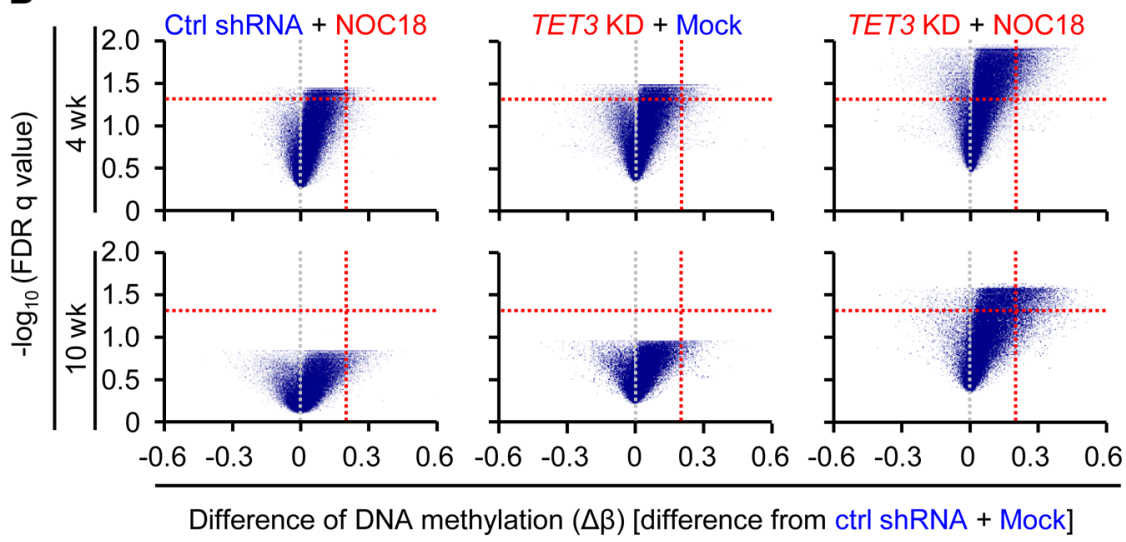

Figure 4. Induction of aberrant DNA methylation by a combination of TET3 repression and increased DNMT activity. (A) DNA methylation analysis of 293FT cells with TET3 knockdown (KD) alone, NOC18 treatment alone, and their combination. TET3 knockdown alone induced aberrant DNA methylation at only a small number of genomic blocks. NOC18 treatment alone induced aberrant methylation at a minimal number of genomic blocks. In contrast, their combination induced aberrant DNA methylation at a large number of genomic blocks. The number of methylated genomic blocks by the combination markedly increased in a culture period-dependent manner. Genomic blocks with $\Delta \beta$ value $\geq 0.2$ are in triangles with a red broken line, and their numbers are noted. The data obtained from 1 of the 3 independent cultures are shown. (B) Volcano plot analysis of DNA methylation differences. The number of genomic blocks with larger $\Delta \beta$ values $(\Delta \beta \geq 0.2)$ and larger $-\log _{10}(F D R q)$ values $(\geq 1.3)$ greatly increased in $293 \mathrm{FT}$ cells treated with the combination. Red lines show $\Delta \beta$ value of 0.2 , and $-\log _{10}(\operatorname{FDR} q)$ value of $1.3(q<0.05)$.

methylation data were submitted to the Gene Expression Omnibus (GEO) database under accession no. GSE117528.

Gene expression analysis. Total RNA was extracted using ISOGEN (Nippon Gene). From $3 \mu \mathrm{g}$ total RNA, cDNA was synthesized using SuperScript III reverse transcriptase (Thermo Fisher Scientific). Genome-wide gene expression analysis was conducted using a SurePrint G3 Human Gene Expression 8x60K v2 Microarray (Agilent
Technologies) as described (52). Gene expression analysis of specific genes was conducted by quantitative RT-PCR (qRT-PCR) as described (53), using primers listed in Supplemental Table 4. The copy number of cDNA molecules of an individual gene was normalized to that of an internal control gene.

Analysis of the 5-hmC content. Genomic DNA $(1 \mu \mathrm{g})$ was denatured at $100^{\circ} \mathrm{C}$ and cooled on ice. Then, DNA was digested with $2 \mathrm{U}$ nuclease P1 (Wako Chemical), followed by treatment with $0.1 \mathrm{U}$ venom phosphodiesterase I (Worthington Biochemical) and $10 \mathrm{U}$ calf intestinal alkaline phosphatase (New England Biolabs) (54). The amounts of 6 deoxyribonucleosides [2'-deoxyguanosine (dG), 2'-deoxyadenosine (dA), $2^{\prime}$-deoxycytidine (dC), 2'-deoxythymidine (dT), 5-methyl-2'-deoxycytidine (5-mC), and 5-hmC] in the hydrolyzed DNA samples were analyzed by the LC/MS/MS system of API2000 (AB SCIEX) equipped with the Shimadzu 10ADvp HPLC system (Shimadzu). The global 5-hmC content was calculated as the fraction of $5-\mathrm{hmC}$ in the total dC (sum of dC, 5-mC, and 5-hmC).

Expression analysis of miRNAs and in silico prediction of Tet-targeting miRNAs. Genome-wide analysis of miRNA expression was performed using a mouse miRNA microarray, Release 21.0, 8x60K (Agilent Technologies). Briefly, $100 \mathrm{ng}$ total RNA was dephosphorylated by calf intestine alkaline phosphatase and was incubated at $100^{\circ} \mathrm{C}$, followed by cooling on ice. Denatured RNA was labeled with Cyanine3, purified by a MicroBioSpin6 column (Bio-Rad), and hybridized to a microarray. The microarray was scanned using an Agilent G2565BA Microarray Scanner (Agilent Technologies), and the scanned data were analyzed using Feature Extraction software (Agilent Technologies) and GeneSpring software Ver.12.5 (Agilent Technologies). In silico prediction of Tet-targeting miRNAs was performed using miRanda (microRNA.org).

Introduction of specific miRNAs into cultured cells. A total of $3 \times 10^{5}$ cells of $293 \mathrm{FT}$ or MCF7 were seeded on day 0 , and were transfected with 20 pmol mirVana miRNA mimics (hsa-miR-29c3p, hsa-miR-26b-5p, or hsa-miR-20a-5p; Thermo Fisher Scientific) using Lipofectamine RNAiMAX reagent (Thermo Fisher Scientific) on day 1 . The cells were harvested on day 2 , and expression levels of TET1, TET2, and TET3 were analyzed by qRT-PCR.

Vector construction and luciferase assay. The 3'-untranslated region (UTR) of human TET3 containing MIR26B target sites (Supplemental Figure 9) was amplified using primers listed in Supplemental Table 5. PCR products were digested with XhoI and SalI restriction enzymes, and were cloned into the pmirGLO DualLuciferase miRNA Target Expression Vector (Promega). Mutations 
A

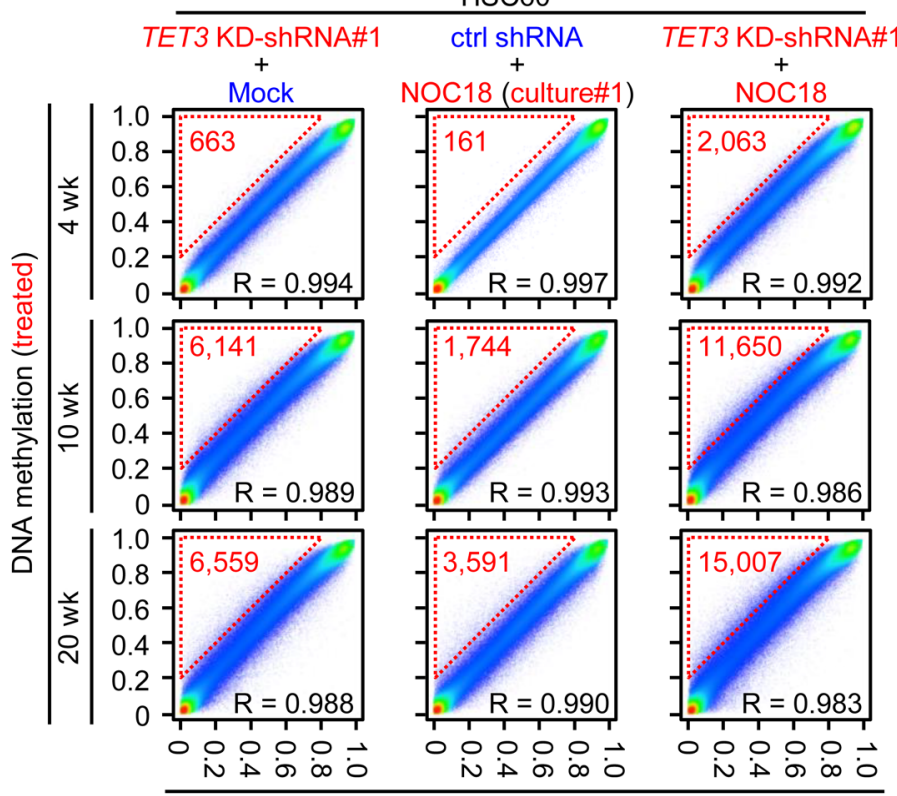

B

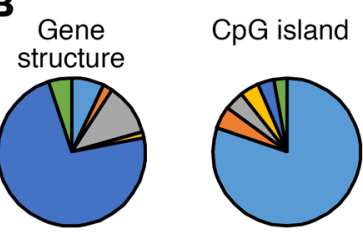

Methylated by the combination
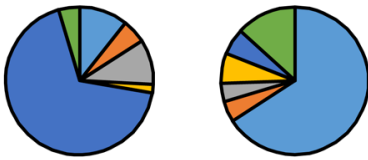

All blocks

$\begin{array}{ll}\square \text { TSS1500 } & \square \text { Non-CG } \\ \square \text { TSS200 } & \square \text { N shelf } \\ \square \text { 5'-UTR }^{\text {st exon }} & \square \text { shelf } \\ \square \text { shore } \\ \square \text { Body } & \square \text { shore } \\ \square \text { 3'-UTR } & \square \text { CGI }\end{array}$

DNA methylation (ctrl shRNA + mock)

C

\begin{tabular}{|c|c|c|c|c|c|c|c|c|c|c|c|c|}
\hline & \multicolumn{3}{|c|}{ Normal } & \multicolumn{7}{|c|}{ Noncancerous } \\
\hline Gene & 1 & 2 & 3 & 24 & 31 & 37 & 41 & 50 & 51 & 52 & 54 & 62 \\
\hline ACP5 & & & & & & & & & & & & \\
\hline ACVR1C & & & & & & & & & & & & \\
\hline C4orf42 & & & & & & & & & & & & \\
\hline CPT1C & & & & & & & & & & & & \\
\hline FAM132A & & & & & & & & & & & & \\
\hline FFAR1 & & & & & & & & & & \\
\hline ICAM1 & & & & & & & & & & & & \\
\hline RASL12 & & & & & & & & & & & & \\
\hline RNF152 & & & & & & & & & & & & \\
\hline SORCS1 & & & & & & & & & & & & \\
\hline SYT14 & & & & & & & & & & & & \\
\hline UNC80 & & & & & & & & & & & \\
\hline WDR86 & & & & & & & & & & & & \\
\hline
\end{tabular}

Unmethylated

Partially methylated

Methylated
D

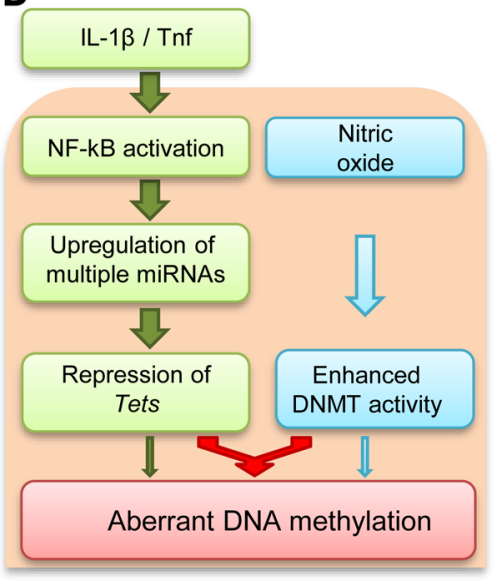

Figure 5. Biological relevance of methylation induction by the combination of TET repression and NOC18 treatment. (A) Synergistic effect of a combination of TET3 knockdown (KD) and NOC18 treatment confirmed in a gastric cancer cell line, HSC60. Compared with TET3 knockdown alone or NOC18 treatment alone, their combination strongly induced aberrant DNA methylation in the HSC60 cells in a culture period-dependent manner. Genomic blocks with $\Delta \beta$ value $\geq 0.2$ are in triangles with a red broken line, and their numbers are noted. (B) Characteristics of genomic regions aberrantly methylated by the combination. Most of the hypermethylated genomic blocks were located in gene body regions without CpG islands. (C) Biological relevance of genomic regions aberrantly methylated by the combination. Genes methylated by the combination in HSC60 were also methylated in primary noncancerous tissues of gastric cancer patients. (D) A model of induction of aberrant DNA methylation by chronic inflammation. In biological settings, chronic inflammation can induce both TET repression and increased DNMT activity. This vicious combination was considered to cooperatively induce aberrant DNA methylation, even in genomic regions resistant to DNA methylation induction.

of MIR26B target sites were introduced using primers listed in Supplemental Table 5. Sequences of the constructed vectors were confirmed by dideoxy sequencing.

For luciferase assay, 100 ng pmirGLO Dual-Luciferase miRNA Target Expression Vector containing the 3 '-UTR sequence was transfected into the 293FT cells in the presence or absence of $10 \mathrm{nM}$ of mirVana miRNA Mimics. At 48 hours after transfection, activities of firefly luciferase and Renilla luciferase were measured using the Dual-Glo Luciferase Assay System (Promega) and an ARVO MX 1420 multilabel counter (PerkinElmer).
Chromatin immunoprecipitation sequencing (ChIP-seq). Crosslinked chromatin $(100 \mu \mathrm{g})$ extracted from NUGC-3 cells with mock and TNF- $\alpha(30 \mathrm{ng} / \mathrm{mL})$ treatment was immunoprecipitated using 5 $\mu \mathrm{g}$ antibody against RELA (R\&D Systems, catalog AF5078). Immunoprecipitated and input DNA were end-repaired to generate $3^{\prime}$-dA overhangs, and adapters were ligated to each end using the NEBNext Ultra II DNA Library Prep Kit (New England Biolabs). DNA fragments with sizes ranging from $100 \mathrm{bp}$ to $600 \mathrm{bp}$ were selected by Agencourt AMPure XP (Beckman Coulter) after 15 cycles of PCR amplification, and were sequenced using the Illumina HiSeq 4000 
in $150 \mathrm{bp}$ pair-end mode at a final sequencing depth of 36 to 47 million reads per sample.

ChIP-seq data were aligned to the hg19 version of the human reference genome using bowtie2 (v2.4.1) (55) with the following parameters: -D15 -R2 -NO - L22 -I s, 1, 1.15 -x hg19. Peaks were called by comparison to the background data (input DNA) using MACS2 (v2.1.0) using the narrowpeak mode and with the following parameters: $-\mathrm{g}$ hs -q 1e-6 -f BAMPE -B (56). Fragment pileup at every base pair was normalized to reads per million mapped reads, and was displayed with the location of peaks in the Integrative Genomics Viewer (57). Heatmap of ChIP/input enrichment around TSS (from $-2.5 \mathrm{~kb}$ to $+2.5 \mathrm{~kb}$ ) was obtained using DROMPA3 (v3.7.1) with the following parameters: -stype 1 -scale_ratio 2 (58).

Enrichment of transcription factor binding motifs was analyzed for 200 bp regions around identified peak summits in TNF- $\alpha$-treated NUGC-3 cells using HOMER findMotifsGenome.pl command with the following parameters: -size 200 hg19r (59).

Western blotting. Western blotting was conducted as described (52), using a rabbit monoclonal antibody against NF-кB p65 (1:1000; catalog 8242; Cell Signaling Technology Japan), rabbit monoclonal antibody against phospho-NF-кB p65 (Ser536) (1:1000; catalog 3033; Cell Signaling Technology Japan), and a goat polyclonal antibody against Actin (1:200; sc-1616; Santa Cruz Biotechnology).

Measurement of DNA methyltransferase activity. Nuclear proteins were extracted from gastric cancer cell lines (HSC41 and TMK1) by an EpiQuik Nuclear Extraction Kit I (Epigentek). Using $10 \mu$ g nuclear protein, DNMT activity was measured by an EpiQuik DNMT Activity/Inhibition Assay Ultra Kit (Epigentek), which can measure total DNMT activity (both de novo and maintenance activities).

Knockdown of TET3 by shRNA. TET3 was knocked down by 3 independent shRNAs as described (60). Briefly, the sense and antisense oligonucleotides containing shRNA sequence (A-022722-13, A-022722-14, and A-022722-16; Horizon Discovery) were annealed. Annealed DNA was cloned into pGreenPuro shRNA Cloning and Expression Lentivector (System Biosciences), and the constructed vector was packaged into lentivirus. 293FT cells or HSC60 cells were infected with lentivirus, and cells with stable expression of shRNA were obtained by puromycin selection.

Nitric oxide treatment. NOC18 (Dojindo Laboratories) and SNAP ( $S$-nitroso- $N$-acetyl-DL-penicillamine) (MilliporeSigma Japan) were used as NO donors. Cells with TET3 knockdown or control cells were seeded $\left(3 \times 10^{4}\right.$ cells) on day 0, and were treated with NOC18 for 6 days (from days 1 to 7 ). Then, cells were trypsinized, and $3 \times 10^{4}$ cells were reseeded. Six-day treatment of NOC18 was repeated 19 times (total $=20$ times).

Gene ontology analysis. Gene ontology analysis was performed by DAVID bioinformatics resources $6.8(61,62)$. The enrichment of specific biological processes (category, GOTERM_BP_ALL) in genes hypermethylated by the combination but not by TET3 knockdown alone or NOC18 treatment alone among all the genes with promoter $\mathrm{CpG}$ islands was analyzed.

Statistics. The difference of gene expression levels and fraction of 5-hmC were evaluated by the Welch's $t$ test (2-tailed); $P$ values less than 0.05 were considered significant. Correlation coefficient $(r)$ and $P$ values were calculated by Pearson's correlation analysis. FDR $q$ values were calculated using $\mathrm{R}$ with qvalue package.

Study approval. All the animal experiments were approved by the Committee for Ethics in Animal Experimentation at the National Cancer Center. The study using clinical samples was approved by the Institutional Review Boards of the National Cancer Center (2012-305) and Toyama University (Rin29-11).

\section{Author contributions}

HT and TU conceived the experimental plan. HT, TN, SY, TTE, MW, and MA carried out the experiments. HT, NI, YJK, and TU conducted data analysis. SN and TS collected clinical samples. HT and TU wrote the manuscript.

\section{Acknowledgments}

This work was supported by Japan Agency for Medical Research and Development grant JP19ck0106267; Japan Society for the Promotion of Science KAKENHI grant JP15H04302; The Naito Foundation; the National Cancer Center Research and Development Fund Japan (26-A-15); and the Ministry of Science ICT Korea (grant 2016M3C9A4921712). We thank T. Imai and the National Cancer Center Research Core Facility for preparation of mouse tissue sections.

Address correspondence to: Toshikazu Ushijima, 5-1-1 Tsukiji, Chuo-ku, Tokyo 104-0045, Japan. Phone: 81.3.3547.5240; Email: tushijim@ncc.go.jp.
1. Baylin SB, Jones PA. A decade of exploring the cancer epigenome-biological and translational implications. Nat Rev Cancer. 2011;11(10):726-734.

2. Esteller M. Cancer epigenomics: DNA methylomes and histone-modification maps. Nat Rev Genet. 2007;8(4):286-298.

3. Timp W, Feinberg AP. Cancer as a dysregulated epigenome allowing cellular growth advantage at the expense of the host. Nat Rev Cancer. 2013;13(7):497-510.

4. Hwang JY, Aromolaran KA, Zukin RS. The emerging field of epigenetics in neurodegeneration and neuroprotection. Nat Rev Neurosci. 2017;18(6):347-361.

5. Barrès R, Zierath JR. The role of diet and exercise in the transgenerational epigenetic landscape of T2DM. Nat Rev Endocrinol. 2016;12(8):441-451. 6. Miranda-Duarte A. DNA methylation in osteo- arthritis: current status and therapeutic implications. Open Rheumatol J. 2018;12:37-49.

7. Gonzalez-Zulueta M, et al. Methylation of the 5' CpG island of the p16/CDKN2 tumor suppressor gene in normal and transformed human tissues correlates with gene silencing. Cancer Res. 1995;55(20):4531-4535.

8. Machado JC, et al. E-cadherin gene (CDH1) promoter methylation as the second hit in sporadic diffuse gastric carcinoma. Oncogene. 2001;20(12):1525-1528.

9. Ohtani-Fujita N, et al. Hypermethylation in the retinoblastoma gene is associated with unilateral, sporadic retinoblastoma. Cancer Genet Cytogenet. 1997;98(1):43-49.

10. Rice JC, Massey-Brown KS, Futscher BW. Aberrant methylation of the BRCA1 CpG island promoter is associated with decreased BRCA1
mRNA in sporadic breast cancer cells. Oncogene. 1998;17(14):1807-1812.

11. Takeshima H, Niwa T, Toyoda T, Wakabayashi M, Yamashita S, Ushijima T. Degree of methylation burden is determined by the exposure period to carcinogenic factors. Cancer Sci. 2017;108(3):316-321.

12. Ushijima T. Epigenetic field for cancerization. J Biochem Mol Biol. 2007;40(2):142-150.

13. Ushijima T, Hattori N. Molecular pathways: involvement of Helicobacter pylori-triggered inflammation in the formation of an epigenetic field defect, and its usefulness as cancer risk and exposure markers. Clin Cancer Res. 2012;18(4):923-929.

14. Nakajima T, et al. Higher methylation levels in gastric mucosae significantly correlate with higher risk of gastric cancers. Cancer Epidemiol 
Biomarkers Prev. 2006;15(11):2317-2321.

15. Asada K, et al. Demonstration of the usefulness of epigenetic cancer risk prediction by a multicentre prospective cohort study. Gut. 2015;64(3):388-396.

16. Maeda M, et al. High impact of methylation accumulation on metachronous gastric cancer: 5 -year follow-up of a multicentre prospective cohort study. Gut. 2017;66(9):1721-1723.

17. Issa JP, Ahuja N, Toyota M, Bronner MP, Brentnall TA. Accelerated age-related $\mathrm{CpG}$ island methylation in ulcerative colitis. Cancer Res. 2001;61(9):3573-3577.

18. Issa JP, Ottaviano YL, Celano P, Hamilton SR, Davidson NE, Baylin SB. Methylation of the oestrogen receptor $\mathrm{CpG}$ island links ageing and neoplasia in human colon. Nat Genet.1994;7(4):536-540.

19. Maegawa S, et al. Widespread and tissue specific age-related DNA methylation changes in mice. Genome Res. 2010;20(3):332-340.

20. Ushijima T, Takeshima H. Epigenetic epidemiology of infectious diseases. In: Michels, KB, ed. Epigenetic Epidemiology. Springer Science; 2011:269-288.

21. O'Hagan HM, et al. Oxidative damage targets complexes containing DNA methyltransferases, SIRT1, and polycomb members to promoter $\mathrm{CpG}$ Islands. Cancer Cell. 2011;20(5):606-619.

22. Cheng AS, et al. Epithelial progeny of estrogenexposed breast progenitor cells display a cancer-like methylome. Cancer Res. 2008;68(6):1786-1796.

23. Hsu PY, et al. Estrogen-mediated epigenetic repression of large chromosomal regions through DNA looping. Genome Res. 2010;20(6):733-744.

24. Oka D, et al. The presence of aberrant DNA methylation in noncancerous esophageal mucosae in association with smoking history: a target for risk diagnosis and prevention of esophageal cancers. Cancer. 2009;115(15):3412-3426.

25. Maekita T, et al. High levels of aberrant DNA methylation in Helicobacter pylori-infected gastric mucosae and its possible association with gastric cancer risk. Clin Cancer Res. 2006;12(3 Pt 1):989-995.

26. Niwa T, et al. Inflammatory processes triggered by Helicobacter pylori infection cause aberrant DNA methylation in gastric epithelial cells. Cancer Res. 2010;70(4):1430-1440.

27. Kondo Y, Kanai Y, Sakamoto M, Mizokami M, Ueda R, Hirohashi S. Genetic instability and aberrant DNA methylation in chronic hepatitis and cirrhosis--A comprehensive study of loss of heterozygosity and microsatellite instability at 39 loci and DNA hypermethylation on $8 \mathrm{CpG}$ islands in microdissected specimens from patients with hepatocellular carcinoma. Hepatology. 2000;32(5):970-979.

28. Okamoto Y, et al. Hepatitis virus infection affects DNA methylation in mice with humanized livers. Gastroenterology. 2014;146(2):562-572.

29. Jusakul A, et al. Whole-genome and epigenomic landscapes of etiologically distinct subtypes of cholangiocarcinoma. Cancer Discov. 2017;7(10):1116-1135.
30. Hur K, et al. Insufficient role of cell proliferation in aberrant DNA methylation induction and involvement of specific types of inflammation. Carcinogenesis. 2011;32(1):35-41.

31. Katsurano M, et al. Early-stage formation of an epigenetic field defect in a mouse colitis model, and non-essential roles of T- and B-cells in DNA methylation induction. Oncogene. 2012;31(3):342-351.

32. Han L, et al. AJAP1 is dysregulated at an early stage of gliomagenesis and suppresses invasion through cytoskeleton reorganization. CNS Neurosci Ther. 2014;20(5):429-437.

33. Namba-Fukuyo $\mathrm{H}$, et al. TET2 functions as a resistance factor against DNA methylation acquisition during Epstein-Barr virus infection. Oncotarget. 2016;7(49):81512-81526.

34. Takayama K, et al. TET2 repression by androgen hormone regulates global hydroxymethylation status and prostate cancer progression. Nat Com mun. 2015;6:8219.

35. Chang TC, et al. Widespread microRNA repression by Myc contributes to tumorigenesis. Nat Genet. 2008;40(1):43-50.

36. Huang G, Nishimoto K, Zhou Z, Hughes D, Kleinerman ES. miR-20a encoded by the miR-1792 cluster increases the metastatic potential of osteosarcoma cells by regulating Fas expression. Cancer Res. 2012;72(4):908-916.

37. Gantier MP, et al. Analysis of microRNA turnover in mammalian cells following Dicer1 ablation. Nucleic Acids Res. 2011;39(13):5692-5703.

38. Hmadcha A, Bedoya FJ, Sobrino F, Pintado E. Methylation-dependent gene silencing induced by interleukin 1beta via nitric oxide production. J Exp Med.1999;190(11):1595-1604.

39. Huang FY, Chan AO, Rashid A, Wong DK, Cho CH, Yuen MF. Helicobacter pylori induces promoter methylation of E-cadherin via interleukin- $1 \beta$ activation of nitric oxide production in gastric cancer cells. Cancer. 2012;118(20):4969-4980.

40. Bibikova M, et al. High density DNA methylation array with single $\mathrm{CpG}$ site resolution. Genomics. 2011;98(4):288-295.

41. Dedeurwaerder S, Defrance M, Calonne E, Denis $\mathrm{H}$, Sotiriou C, Fuks F. Evaluation of the infinium methylation $450 \mathrm{~K}$ technology. Epigenomics. 2011;3(6):771-784.

42. Iida N, Okuda Y, Ogasawara O, Yamashita S, Takeshima H, Ushijima T. MACON: a web tool for computing DNA methylation data obtained by the Illumina Infinium Human DNA methylation BeadArray. Epigenomics. 2018;10(3):249-258.

43. Eads CA, et al. Fields of aberrant CpG island hypermethylation in Barrett's esophagus and associated adenocarcinoma. Cancer Res. 2000;60(18):5021-5026.

44. Amor S, et al. Inflammation in neurodegenerative diseases--an update. Immunology. 2014;142(2):151-166.

45. Ray I, Mahata SK, De RK. Obesity: An immunometabolic perspective. Front Endocrinol (Lausanne). 2016;7:157

46. Sellam J, Berenbaum F. The role of synovitis in pathophysiology and clinical symptoms of osteoarthritis. Nat Rev Rheumatol. 2010;6(11):625-635.

47. Robertson KD. DNA methylation and human disease. Nat Rev Genet. 2005;6(8):597-610.

48. Yoshida T, et al. Alu and Sat $\alpha$ hypomethylation in Helicobacter pylori-infected gastric mucosae. Int J Cancer. 2011;128(1):33-39.

49. Bariol C, et al. The relationship between hypomethylation and $\mathrm{CpG}$ island methylation in colorectal neoplasia. Am J Pathol. 2003;162(4):1361-1371.

50. Cheng H, Bjerknes M, Amar J. Methods for the determination of epithelial cell kinetic parameters of human colonic epithelium isolated from surgical and biopsy specimens. Gastroenterology. 1984;86(1):78-85

51. Yoda Y, et al. Integrated analysis of cancer-related pathways affected by genetic and epigenetic alterations in gastric cancer. Gastric Cancer. 2015;18(1):65-76.

52. Takeshima H, Wakabayashi M, Hattori N, Yamashita S, Ushijima T. Identification of coexistence of DNA methylation and H3K27me3 specifically in cancer cells as a promising target for epigenetic therapy. Carcinogenesis. 2015;36(2):192-201.

53. Takeshima H, Yamashita S, Shimazu T, Niwa T, Ushijima T. The presence of RNA polymerase II, active or stalled, predicts epigenetic fate of promoter CpG islands. Genome Res. 2009;19(11):1974-1982.

54. Friso S, Choi SW, Dolnikowski GG, Selhub J. A method to assess genomic DNA methylation using high-performance liquid chromatography/ electrospray ionization mass spectrometry. Anal Chem. 2002;74(17):4526-4531.

55. Langmead B, Salzberg SL. Fast gappedread alignment with Bowtie 2. Nat Methods. 2012;9(4):357-359.

56. Zhang Y, et al. Model-based analysis of ChIP-Seq (MACS). Genome Biol. 2008;9(9):R137.

57. Robinson JT, et al. Integrative genomics viewer. Nat Biotechnol. 2011;29(1):24-26.

58. Nakato R, Itoh T, Shirahige K. DROMPA: easyto-handle peak calling and visualization software for the computational analysis and validation of ChIP-seq data. Genes Cells. 2013;18(7):589-601.

59. Heinz S, et al. Simple combinations of lineagedetermining transcription factors prime cis-regulatory elements required for macrophage and $\mathrm{B}$ cell identities. Mol Cell. 2010;38(4):576-589.

60. Kikuyama M, et al. Development of a novel approach, the epigenome-based outlier approach, to identify tumor-suppressor genes silenced by aberrant DNA methylation. Cancer Lett. 2012;322(2):204-212.

61. Huang da W, Sherman BT, Lempicki RA. Systematic and integrative analysis of large gene lists using DAVID bioinformatics resources. Nat Protoc. 2009;4(1):44-57.

62. Huang da W, Sherman BT, Lempicki RA. Bioinformatics enrichment tools: paths toward the comprehensive functional analysis of large gene lists. Nucleic Acids Res. 2009;37(1):1-13. 